\title{
Incompleteness of General Relativity Regarding Einstein's Program
}

\author{
Claude Elbaz ${ }^{1}$ \\ ${ }^{1}$ Academie Europeenne Interdisciplinaire de Science (A.E.I.S.), Paris, France \\ Correspondence: Claude Elbaz, Academie Europeenne Interdisciplinaire de Science (A.E.I.S.), Paris, France. \\ E-mail: claude.elbaz@science-inter.com
}

Received: July 5, 2018

doi:10.5539/apr.v10n5p47
Accepted: August 29, 2018

Online Published: September 27, 2018

URL: https://doi.org/10.5539/apr.v10n5p47

\begin{abstract}
The detection of gravitational waves substantiates the undeniable achievement of general relativity theory by increasing its theoretical and experimental accuracy. One century after predicting it has set again Einstein's works at the front of research. Absence of quantum particle associated to gravitation emphasizes that general relativity theory remains not included in the standard model of physics. Then Einstein's disagreement about it incompleteness regarding wave-particle and matter-field becomes actualized. In order to circumvent these difficulties he privileged field, rather than matter for universe description in his program. In consequence a scalar field $\varepsilon\left(\mathrm{r}_{0}, \mathrm{t}_{0}\right)$ propagating at speed of light $\mathrm{c}$ yields matter from standing waves moving at speed strictly inferior to $\mathrm{c}$, and interactions from progressive waves. Electromagnetic interactions derive from local variations of frequencies, and gravitation from local variations of speed of light. A space-like amplitude functions $\mathrm{u}_{0}\left(\mathrm{k}_{0} \mathrm{r}_{0}\right)$ supplements fundamental time-like functions of classical and quantum mechanics. It tends toward Dirac's distribution $\delta\left(\mathrm{r}_{0}\right)$ in geometrical optics approximation conditions, when frequencies are infinitely high, and then hidden.

More generally, it allows theoretical economies by deriving energy-momentum conservation laws, and least action law. Quantum domain corresponds to wave optics approximation conditions. Variations of frequencies give rise to an adiabatic constant, formally identical with Planck's constant, leading to first quantification for electromagnetic interactions and to second quantification for matter.
\end{abstract}

Keywords: Einstein's program, General Relativity Theory, Adiabatic Invariant, Planck's constant, Wave-Particle duality

\section{Introduction}

The last and most recent important discovery in physics was the first direct experimental detection of gravitational waves in 2016, one century after their prediction by Einstein's equations of General Relativity. It was largely reported by world mass media. As a mark of importance for the scientific community, the Nobel's prize was promptly attributed of to the contributors in 2017.

Two main features of the results were usually pointed out: the physical wave character of gravitation and the smallness of the energy detected. Both enhanced the mathematical accuracy of the Einstein's equations, and the physical validity of the theory. They confirm not only that General Relativity is not superseded upon exploration of universe, but that gravitational waves offer now means to forge a new kind of astronomy, in addition to usual electromagnetic waves.

Then, after one century of dedicated efforts, carried on by many physicists in the world, Einstein's works came into view again at the front of research. We are then incited to reexamine reasons of his disagreement with the majority of physicists of his time: they most largely admitted the quantum probabilistic approach, owing to the numerous successful results obtained from theory, experiment, and associated technology which led to the Standard Model of Particles. It gathers almost the whole present knowledge upon theoretical physics, except gravitation which has still resisted to its quantification. In a consistent system, the Standard Model describes the universe as constituted fundamentally of particles, both for matter and for interactions.

The experimental detection of gravitational waves emphasized the incompleteness of the Standard Model with regard to gravitation in two ways. In one hand, gravitation is not included as a fundamental interaction beside the three others. In another hand, no graviton, as mediating particle, was associated. The detected wave behavior derived directly from the experimental interferometric method, which is specifically used to discriminate waves 
from particles. But symmetrically, the experimental detection of gravitational waves emphasized also the incompleteness of the General Relativity equations regarding quantification and wave-particle duality.

With regard to quantification, the classical deterministic equations of General Relativity were established in 1915 before the development of the probabilistic approach of the quantum theory, resolutely opposed by Einstein afterward. In order to appreciate the preeminence he gave to the classical and deterministic approach, we notice that, almost simultaneously with his publications on relativity theories in years 1905 and 1916, he brought major contributions to quantum theory. This shows that classical and quantum properties where not dissociated in his mind. In 1905, concurrently with Special Relativity, he introduced the first quantum particle for light with energy quanta $E=h v$, afterward designated as photon, from the photoelectric effect. In 1916, concurrently with General Relativity, he discovered the quantum stimulated emission of light in atoms (Einstein, 1916). For his contemporary physicists, the Einstein's contribution to quantum theory was more important, since he the Nobel's prize awarded him in 1921 for his introduction of the light quantum particle, and not for Special and General Relativity theories.

With regard to wave-particle duality, Einstein expressed his lack of entire satisfaction by the General Relativity theory, despite his awareness about its achievement: it was incomplete because of the coexistence of localized particles and extended fields in its equations. "One would be compelled to demand that the particles themselves would everywhere be described as singularity-free solutions of the completed field-equations. Only then would the general theory of relativity be a complete theory." He noticed that "The theory of relativity stresses the importance of the field concept in physics. But we have not yet succeeded in formulating a pure field physics. For the present we must still assume the existence of both: field and matter." (Einstein,1949). Since its equations contained the particles of matter in the right size and the gravitation field described with higher precision the left size, he used the comparison: " general theory of relativity is similar to a building, one wing of which is made of fine marble, but the other wing of which is built of low grade wood."

In order to remedy, his successive significant discoveries convinced him that a classical extended field would play a more basic role in a unified approach of the constitution of universe. In his program, he noticed: «We have two realities: matter and field. .... We cannot build physics on the basis of the matter concept alone. But the division into matter and field is, after the recognition of the equivalence of mass and energy, something artificial and not clearly defined. Could we not reject the concept of matter and build a pure field physics? ...We could regard matter as the regions in space where the field is extremely strong. In this way a new philosophical background could be created.... Only field-energy would be left, and the particle would be merely an area of special density of field-energy. In that case one could hope to deduce the concept of the mass-point together with the equations of the motion of the particles from the field equations- the disturbing dualism would have been removed... One would be compelled to demand that the particles themselves would everywhere be describable as singularity free solutions of the completed field-equations. Only then would the general theory of general relativity be a complete theory.... One could believe that it would be possible to find a new and secure foundation for all physics upon the path which had been so successfully begun by Faraday and Maxwell.

Accordingly, the revolution begun by the introduction of the field was by no means finished» (Einstein, 1938).

Then, beyond the problem of completing General Relativity theory with regard to wave-particle duality, the program tends to a broader unified approach, since, according to Einstein " $A$ theory is the more impressive the greater the simplicity of its premises is, the more different kinds of things it relates, and the more extended is its area of applicability."

Guided by the program, its development raises many questions about thought representation, experiment, physical model, mathematical theory, and technology.

About thought representation, it is well known that Einstein was accustomed to give priority to physical models for representations, and to rely upon thoughts experiments, like for some famous controversies with Bohr. We find such physical models for representations and physical thoughts experiments, at the basis of his theories of Relativity: with the reciprocal electrodynamic motion and interaction of a magnet and a conductor, at origin of Special Relativity, and with the equivalence of gravitation with accelerated motion of bodies in free fall, for General Relativity. In both cases, the geometrical and mathematical abstract properties of the space-time occurred afterward.

From an experimental point of view, we notice that, since the 1960 years, the Einstein's Program has been implicitly validated and implemented by the International Legal Metrology Organization when matter was replaced by field, in order to determine the standards for measures of length and time, after having served as a fundamental basis during two centuries. Nowadays the standards are based upon one particular period of an 
electromagnetic wave frequency (Jefferts \& al, 2015). The velocity of light in vacuum is admitted as a primary fundamental constant in physics, with its numerical value strictly fixed. By comparison, the Planck's constant is not yet considered as a primary fundamental constant for the determination of the standard for measures of mass, which is still based on matter.

About physical models, owing to the prominent role of experiment and measure in physics, the international standards for measures of length and of time lead us to banish rigid rods and material clocks, for representations of matter behaviors in space and time, on behalf of waves propagating at light velocity. More especially as electronic devices, like electronic telemeters or numerical clocks, are largely more precise than material devices.

From a technological point of view, more generally, and even implicitly, the Einstein's Program is largely and progressively implemented, when we notice that, almost systematically, all usual material devices are replaced by numerical and electromagnetic devices.

From a theoretical point of view, following the Einstein's program, we propose to show how the General Relativity may be completed with regard to wave-particle duality by privileging the field. More generally, it paves the way towards unification of physics by deriving some classical and quantum properties of matter and interactions. For this purpose, a scalar field $\varepsilon(r, t)$ propagating at speed of light forms a consistent system for universe description.

\section{Properties of the C-Scalar Field}

We restrict to summarize some equations for the properties of the c-scalar field, according to Einstein's program, previously published, (Elbaz, 2010, 2012, 2013, 2014), in order to show how they are related to some main equations of classical and quantum mechanics, otherwise widely documented.

\subsection{Kinematical Properties of Standing Fields}

Starting from the d'Alembertian's equation describing a scalar field $\varepsilon$ propagating at constant light velocity c, we make sure that all following results hold in the special relativist framework

$$
\varepsilon=\Delta \varepsilon-\left(1 / \mathrm{c}^{2}\right)\left(\partial^{2} \varepsilon / \partial \mathrm{t}^{2}\right)=0, \quad \partial^{\mu} \partial_{\mu} \varepsilon=0
$$

It admits two kinds of elementary harmonic solutions with constant frequency $\omega_{0}$, with different kinematical properties: progressive and standing waves. Oscillating progressive waves, either retarded like $\cos \left(\omega_{0} \mathrm{t}_{0}-\mathrm{k}_{0} \mathrm{x}_{0}\right)$ or advanced like $\cos \left(\omega_{0} \mathrm{t}_{0}+\mathrm{k}_{0} \mathrm{x}_{0}\right)$, are in motion at light velocity $\mathrm{c}=\omega_{0} / \mathrm{k}_{0}$. Standing waves, with separated space and time variables, $\varepsilon_{0}\left(\mathrm{x}_{0}, \mathrm{t}_{0}\right)=\mathrm{u}_{0}\left(\mathrm{k}_{0} \mathrm{x}_{0}\right) \psi_{0}\left(\omega_{0} \mathrm{t}_{0}\right)=\cos \left(\omega_{0} \mathrm{t}_{0}\right) \cos \left(\mathrm{k}_{0} \mathrm{x}_{0}\right)$, oscillate locally. They allow to define a system of coordinates at rest $\left(\mathrm{x}_{0}, \mathrm{t}_{0}\right)$.

They may be considered as resulting from superposition of two progressive waves

$$
\cos \left(\omega_{0} \mathrm{t}_{0}+\mathrm{k}_{0} \mathrm{x}_{0}\right)+\cos \left(\omega_{0} \mathrm{t}_{0}-\mathrm{k}_{0} \mathrm{x}_{0}\right)=2 \cos \left(\omega_{0} \mathrm{t}_{0}\right) \cos \left(\mathrm{k}_{0} \mathrm{x}_{0}\right)
$$

When, in a system of reference $(\mathrm{x}, \mathrm{t})$, the frequencies of opposite progressive waves are different

$$
\cos \left(\omega_{1} t-k_{1} x\right)+\cos \left(\omega_{2} t+k_{2} x\right)=2 \cos (\omega t-\beta k x) \cos (k x-\beta \omega t)
$$

where $\omega=\left(\omega_{1+} \omega_{2}\right) / 2=\mathrm{kc}$, and $\beta=\left(\omega_{1}-\omega_{2} / \omega_{1+} \omega_{2}\right)$. By identification with $(2)$, they form a standing wave in motion with a speed $\mathrm{v}=\beta \mathrm{c}=\left(\omega_{1}-\omega_{2} / \omega_{1+} \omega_{2}\right)$ c. Its frequency $\omega=\left(\omega_{1+} \omega_{2}\right) / 2=\mathrm{kc}$ corresponds to a rest frequency $\omega_{0}=\sqrt{ } \omega_{1} \omega_{2}$, defining the Lorentz transformation between the systems of reference at rest $\left(\mathrm{x}_{0}, \mathrm{t}_{0}\right)$ and in motion $(\mathrm{x}, \mathrm{t})$, and leading to its whole physical relativist consequences.

It can be shown that the Lorentz transformation, fundamental in special relativity, is specific of c-field standing waves, particularly through the coefficient $\sqrt{ }\left(1-\beta^{2}\right)$ which becomes $(1 \pm \beta)$ for progressive waves (Elbaz, 1984). The four-dimensional Minkowski's formalism expresses invariance properties of standing waves at rest, with separated space and time variables, when they move uniformly with a speed $\mathrm{v}$, necessarily inferior to $\mathrm{c}$, since $\beta$ is a relative difference $v=\beta c<c$. We find confirmation into invariant quantities obtained from four-quantities, such as coordinates $\mathrm{x}_{\mu} \mathrm{x}^{\mu}=\mathrm{x}_{0}{ }^{2}$ or $\mathrm{x}_{\mu} \mathrm{x}^{\mu}=\mathrm{c}^{2} \mathrm{t}_{0}{ }^{2}$, and functions $\mathrm{u}_{\mu} \mathrm{u}^{\mu}=\mathrm{u}^{2}\left(\mathrm{x}_{0}\right)$ or $\psi_{\mu} \psi^{\mu}=\psi^{2}\left(\mathrm{t}_{0}\right)$. Their space-like or time-like characters are absolute, depending of their refering coordinates defined in the rest system.

Since the functions $\mathrm{u}_{0}\left(\mathrm{k}_{0} \mathrm{x}_{0}\right)$ and $\psi_{0}\left(\omega_{0} \mathrm{t}_{0}\right)$ are independent, the frequency $\omega_{0}$ is necessarily constant.

$$
\left(1 / \mathrm{u}_{0}\right) \Delta_{0} \mathrm{u}_{0}=\left(1 / \psi_{0}\right)\left(\partial^{2} \psi_{0} / \mathrm{c}^{2} \partial \mathrm{t}_{0}{ }^{2}\right)=-\mathrm{k}_{0}{ }^{2}=-\omega_{0}{ }^{2} / \mathrm{c}^{2} .
$$

The function of space $\mathrm{u}_{0}\left(\mathrm{k}_{0} \mathrm{x}_{0}\right)$, describing geometrical properties of standing waves obeys the Helmholtz's equation at rest $\Delta_{0} \mathrm{u}_{0}+\mathrm{k}_{0}{ }^{2} \mathrm{u}_{0}=0$, becoming $\Delta \mathrm{u}-\left(1 / \mathrm{c}^{2}\right)\left(\partial^{2} \mathrm{u} / \partial \mathrm{t}^{2}\right)+\mathrm{k}_{0}{ }^{2} \mathrm{u}=0$ in a moving system $(\mathrm{x}, \mathrm{t})$. It verifies Bessel spherical functions solutions, and particularly its simplest elementary solution, with spherical symmetry, finite at origin of the reference system $\left(\mathrm{x}_{0}, \mathrm{t}_{0}\right)$, 


$$
\mathrm{u}_{0}\left(\mathrm{k}_{0} \mathrm{r}_{0}\right)=\left(\operatorname{sink}_{0} \mathrm{r}_{0}\right) /\left(\mathrm{k}_{0} \mathrm{r}_{0}\right)
$$

It leads to Dirac's distribution $\mathrm{u}_{0}\left(\mathrm{k}_{0} \mathrm{r}_{0}\right) \rightarrow \delta\left(\mathrm{r}_{0}\right)$ when frequency tends towards infinity $\omega_{0}=\mathrm{k}_{0} \mathrm{c} \rightarrow \infty$. The central extremum of an extended standing wave determines its position $\mathrm{x}_{0}=\mathrm{r}_{0}=0$. In Cartesian system of reference, it verifies

$$
\nabla_{0} \mathrm{u}_{0}\left(\mathbf{x}_{0}\right)=0 .
$$

In order to point out the constant frequency of a moving standing field, we express it as

$$
\begin{gathered}
\varepsilon(\omega t, k x)=u(k x, \beta c t) \operatorname{exp~i}(\omega t-\beta k x) \\
\varphi=\omega t-\beta k x
\end{gathered}
$$

The equations of special and general relativity are based on mass-points, as singularities derived directly from geometrical optics approximation, moving on trajectories. Consequently, for a scalar field propagating at light velocity $\mathrm{c}$, with constant frequency $\omega$ and constant velocity $\mathrm{v}$, the kinematic properties of standing waves, reduce formally to kinematical properties of isolated point-like matter.

\subsection{Dynamical Properties of Standing Fields}

Since a field $\varepsilon(\omega \mathrm{t}, \mathrm{kx})$ cannot extend physically to infinity with respect to space and time, one imposes usually boundary conditions exerted by matter in order to limit it. Matter behaves either as a source, which fixes the wavelength $\lambda$ through $\mathrm{k}=2 \pi / \lambda$ and then the frequency $\omega=\mathrm{kc}$, or as a detector annealing it. In addition to its heterogeneous character, this is not felicitous from relativistic consistency, since space and time operate separately.

In order to stay in a homogeneous frame, we consider extension in space of two progressive waves with different frequencies $\omega_{1}, \omega_{2}$ propagating in the same direction at light velocity. They give rise to a wave packet propagating in the same direction at light velocity. It forms a main wave with frequency $\omega=\left(\omega_{1+} \omega_{2}\right) / 2$, modulated by a wave with frequency $\beta \omega=\left(\omega_{1}-\omega_{2}\right) / 2=\Delta \omega / 2=\Delta \mathrm{kc} / 2$, a wavelength $\Lambda=2 \pi / \beta \mathrm{k}$, and a period $\mathrm{T}=\Lambda / \mathrm{c}$. Since $\beta<1$, the modulation wave acts as an envelope with space and time extensions $\Delta \mathrm{x}=\Lambda / 2, \Delta \mathrm{t}=\mathrm{T} / 2$, yielding well known Fourier relations $\Delta \mathrm{x} . \Delta \mathrm{k}=2 \pi$ and $\Delta \mathrm{t} . \Delta \omega=2 \pi$.

The homogenous boundary conditions for the scalar field $\varepsilon(\omega \mathrm{t}, \mathrm{kx})$ are represented by the Fourier relations, which must supplement d'Alembertian's equation (1), in order to emphasize that the field cannot extend to infinity with respect to space and time. When the difference of frequencies $\beta \omega$ is very small $\beta \omega=\left(\omega_{1}-\omega_{2}\right) / 2=\Delta \omega / 2<<\omega$, it can be considered as a perturbation $\delta \omega$ with respect to the main frequency, $\beta \omega=\delta \omega$.

An almost monochromatic wave can then be characterized by a frequency $\Omega(\mathrm{x}, \mathrm{t})$ almost constant $\omega$,

$$
\Omega(\mathrm{x}, \mathrm{t})=\mathrm{K}(\mathrm{x}, \mathrm{t}) \mathrm{c}=\omega \pm \delta \Omega(\mathrm{x}, \mathrm{t}) \quad \delta \Omega(\mathrm{x}, \mathrm{t})<<\omega \quad \omega=\text { constant } .
$$

We retrieve the definition of an adiabatic variation for the frequency (Landau, 1960). Consequently, all following properties of almost fields arise inside such a process. In that framework, the necessarily constant frequency of a standing wave must be considered as the mean value, all over the field, of different slowly varying frequencies $\Omega(\mathrm{x}, \mathrm{t})$, instead of a given data. The perturbation frequencies of modulation waves propagating at light velocity $\delta \Omega(\mathrm{x}, \mathrm{t})$ behave then as interactions between main waves, yielding the mean frequency $\omega$ to stay practically constant all over space-time (Elbaz, 2012).

Such a behavior authorizes mathematically to derive the properties of almost fields with varying frequency $\Omega(\mathrm{x}, \mathrm{t})$ from monochromatic constant mean frequency $\omega$, through the variation of constants method (Duhamel principle). Following (8), we express it, as

$$
\varepsilon(\mathrm{x}, \mathrm{t})=\mathrm{U}(\mathrm{x}, \mathrm{t}) \exp \mathrm{i} \phi(\mathrm{x}, \mathrm{t}) \quad \phi(\mathrm{x}, \mathrm{t})=\Omega(\mathrm{x}, \mathrm{t}) \mathrm{t}-\mathbf{K}(\mathrm{x}, \mathrm{t}) \cdot \mathbf{x}+2 \mathrm{n} \pi,
$$

In which products of second order $\delta \Omega \mathrm{dt} \approx 0$ and $\delta \mathbf{K} . \mathrm{d} \mathbf{x} \approx 0$, defined modulo $2 \pi$, are neglected at first order of approximation. This is equivalent to take account that the boundary conditions defined by Fourier relations, are directly incorporated in almost monochromatic solutions,

$$
\mathrm{d} \phi(\mathrm{x}, \mathrm{t})=\mathbf{\Omega}(\mathrm{x}, \mathrm{t}) \mathrm{dt}-\mathbf{K}(\mathrm{x}, \mathrm{t}) \cdot \mathrm{d} \mathbf{x} \approx \omega \mathrm{dt}-\mathbf{k} \cdot \mathrm{d} \mathbf{x} . \quad \mathrm{U}(\mathrm{x}, \mathrm{t})=\mathrm{u}(\mathrm{x}, \mathrm{t}) \pm \delta \mathrm{U}(\mathrm{x}, \mathrm{t})
$$

According to (1) for the field $\varepsilon(\omega t, k x)$, the almost solutions defined by (9) verify,

$$
\begin{gathered}
\partial^{\mu} \partial_{\mu} U-U \partial^{\mu} \phi \partial_{\mu} \phi=0 \\
\partial^{2} U / c^{2} \partial t^{2}-\nabla^{2} U-U\left[(\partial \phi / c \partial t)^{2}-(\nabla \phi)^{2}\right]=0 \\
\partial^{\mu}\left(U^{2} \partial_{\mu} \phi\right)=0 \\
\partial\left(U^{2} \Omega\right) / c^{2} \partial t+\nabla \cdot\left(U^{2} \beta \mathbf{K}\right)=0
\end{gathered}
$$


These relations apply to progressive waves for $\beta= \pm 1$, to standing waves at rest for $\beta=0$ and in motion for $\beta<1$, to monochromatic waves for $\omega$ and $\mathbf{k}$ constant, to almost monochromatic waves for varying $\Omega(\mathrm{x}, \mathrm{t})$ and $\mathbf{K}(\mathrm{x}, \mathrm{t})$. They lead to dynamical properties for energy-momentum conservation, and to least action, principles, for standing fields and for almost standing fields (Elbaz, 1984, 2010, 2014, 2015).

For a standing wave with constant frequency $\omega$, either at rest or in motion, equation (12) reduces to

$$
\begin{gathered}
\partial \mathrm{u}_{0}{ }^{2} / \partial \mathrm{t}_{0}=0 . \quad \partial \mathrm{u}^{2} / \partial \mathrm{t}+\nabla \cdot \mathrm{u}^{2} \mathbf{v}=0 \\
\partial_{\mu} \mathrm{w}^{\mu}=0
\end{gathered}
$$

with $\mathrm{w}^{\mu}=\left(\mathrm{u}^{2}, \mathrm{u}^{2} \mathbf{v} / \mathrm{c}\right)=\mathrm{u}_{0}\left(\mathrm{x}_{0}\right)^{2}(1, \mathbf{v} / \mathrm{c}) / \sqrt{ }\left(1-\beta^{2}\right)$ as a four-dimensional vector. This continuity equation for $\mathrm{u}^{2}$ is formally identical with Newton's equation continuity for matter-momentum density $\partial \mu / \partial t+\nabla$. $\mu \mathbf{v}=0$. By transposition, we can then admit that $u^{2}$ represents the energy density of the standing field.

The centre of amplitude, with position $\mathrm{x}_{0}$ defined by (6), as a characteristic point of a standing field, allows to determine its kinematical behavior. Then, the position $\mathrm{x}_{0}$ of the rest energy density and $\mathrm{x}$ for moving energy density verify

$$
\begin{array}{cl}
\nabla_{0} \mathrm{u}_{0}^{2}=0 & \nabla \mathrm{u}^{2}+\left(\partial \mathrm{u}^{2} \mathbf{v} / \mathrm{c}^{2} \partial \mathrm{t}\right)=0 \quad \nabla \times \mathbf{v}=0 \\
& \pi^{\mu v}=\partial^{\mu} \mathrm{w}^{v}-\partial^{v} \mathrm{w}^{\mu}=0,
\end{array}
$$

The standing wave energy density $u^{2}$, which is spread in space, corresponds to a potential energy density. Then, $\mathbf{F}=-\nabla \mathrm{u}^{2}=-\nabla \mathrm{w}_{\mathrm{P}}$ is a density force, and $\partial \mathrm{u}^{2} \mathbf{v} / \mathrm{c}^{2} \partial \mathrm{t}$ is a density momentum. They are gathered in $\pi^{\mu v}$ as a four-dimensional force density.

By identification of density momentum $u^{2} \mathbf{v} / \mathrm{c}^{2}$ of field and $\mu \mathbf{v}$ of matter, we retrieve the relation of equivalence between mass and energy densities

$$
\begin{gathered}
\mathrm{u}^{2} \mathbf{v} / \mathrm{c}^{2}=\mu \mathrm{v} \\
\mathrm{u}^{2}=\mu \mathrm{c}^{2} .
\end{gathered}
$$

We notice that the light velocity c did not appear in equation of continuity for energy density (13), because it is constant, in the present special relativist framework, and then in the Standard Model. We will show farther that this is no longer true in the general relativity framework of gravitation.

In equation (14), the vanishing four-dimensional force density tensor $\pi^{\mu v}$ of a standing wave, asserts that its space stability at rest holds in motion, and that the energy-momentum density four-vector $\mathrm{w}^{\mu}$ is four-parallel, or directed along the motion velocity $\mathbf{v}$.

Equation (14) is mathematically equivalent to the least action principle, in which energy density $\mathrm{w}^{\mu}$ is a four-dimensional gradient $\partial^{\mu} \mathrm{a}$

$$
\delta \int \mathrm{da}=0 \quad \delta \int \partial^{\mu} \mathrm{adx}_{\mu}=0 \quad \text { with } \quad \mathrm{w}^{\mu}=\partial^{\mu} \mathrm{a} .
$$

When we transpose the mass density $\mu=\mathrm{u}^{2} / \mathrm{c}^{2}$, and we take account of the identities $\nabla \mathrm{P}^{2}=2(\mathbf{P} . \nabla) \mathbf{P}+2 \mathbf{P} \times(\nabla \times \mathbf{P})$ and $\mathrm{d} \mathbf{P} / \mathrm{d} t=\partial \mathbf{P} / \partial \mathrm{t}+(\mathbf{v} . \nabla) \mathbf{P}$ for $\mathrm{c}$ and $\mathrm{v}$ constant, we obtain from (14) the equation for matter after integration with respect to space,

$$
\mathrm{d} \mathbf{p} / \mathrm{dt}=-\nabla \mathrm{mc}^{2}+\left\{\nabla(\mathrm{mv})^{2}\right\} / 2 \mathrm{~m} \quad \mathrm{~d} \mathbf{p} / \mathrm{dt}=\nabla \mathrm{L}_{\mathrm{m}}=-\nabla \mathrm{m}_{0} \mathrm{c}^{2} \sqrt{ }\left(1-\beta^{2}\right) .
$$

We retrieve the relativistic Lagrangian of mechanics for free matter $L_{m}=-m_{0} c^{2} \sqrt{ }\left(1-\beta^{2}\right)$.

Then kinematical and dynamical properties of standing fields are identical with those of free matter.

\subsection{Electromagnetic Interaction}

For an almost standing wave, the continuity equation (12), involves the total energy density, $\mathrm{W}=\mathrm{U}^{2} \Omega=\mathrm{W}+\delta \mathrm{W}$, sum of the mean energy density $w$ of the standing wave with high constant frequency $\omega$, and of the interactions $\delta \mathrm{W}$ with lower varying frequency $\delta \Omega(\mathrm{x}, \mathrm{t})$. The relation (14) becomes

$$
\begin{gathered}
\Pi^{\mu v}=\partial^{\mu} \mathrm{W}^{v}-\partial^{v} \mathrm{~W}^{\mu}=0 \\
\Pi^{\mu v}=\pi^{\mu v}+\delta \Pi^{\mu v}=0
\end{gathered}
$$

The total density force $\Pi^{\mu v}$ for an almost standing wave vanishes. This traduces its stability when in motion. Its total energy-momentum density four-vector $\mathrm{W}^{\mu}$ is directed along the constant moving velocity $\mathrm{v}$.

By difference with the null four-dimensional density force $\pi^{\mu v}$ for a standing wave, only the total density force $\Pi^{\mu v}$ for an almost standing wave vanishes. In the first case, this asserts the space stability of a free, or isolated, moving standing wave, while in the second case, the space stability concerns the whole almost standing wave. It 
behaves as a system composed of two sub-systems, the mean bounded standing field with almost constant high frequency $\Omega(\mathrm{x}, \mathrm{t}) \approx \omega$, and the interaction field with lower frequency $\delta \Omega(\mathrm{x}, \mathrm{t})$. Each one exerts an equal and opposite density force $\pi^{\mu \nu}=-\delta \Pi^{\mu \nu}$ against the other. For the mean bounded standing wave, the energy-momentum density tensor $\pi^{\mu v}$ no longer vanishes in (18), as previously in (14). This comes from the mean energy-momentum density four-vector $\mathrm{w}^{\mu}$, which is no longer parallel, because of the opposite density force $\delta \Pi^{\mu v}$ exerted by the interaction.

It appears that an almost standing field behaves as a whole system in motion, which can be split in two sub-systems, the mean standing field and the interaction field. Both are moving with velocity $\mathrm{v}$, while exerting each other equal opposite forces in different directions, including perpendicularly to the velocity $\mathrm{v}$. The perturbation field, arises from local frequency variations $\delta \Omega(\mathrm{x}, \mathrm{t})$, behaving as interaction. It introduces orthogonal components in interaction density force and momentum.

The relations (17) generalized by constants variation method $\mathrm{M}(\mathrm{x}, \mathrm{t})=\mathrm{m} \pm \delta \mathrm{M}(\mathrm{x}, \mathrm{t})$, becomes

$$
\nabla \mathrm{Mc}^{2}+\partial \mathrm{P} / \partial \mathrm{t}=0 \quad \nabla \times \mathrm{P}=0 \quad \mathrm{dP} / \mathrm{dt}=-\nabla \mathrm{Mc}^{2}+\left(\nabla \mathrm{P}^{2}\right) / 2 \mathrm{M} .
$$

The non vanishing density force $\delta \Pi^{\mu v} \neq 0$ exerted by the interaction, is formally identical with the electromagnetic tensor $\mathrm{F}^{\mu v}=\partial^{\mu} \mathrm{A}^{v}-\partial^{v} \mathrm{~A}^{\mu} \neq 0$. We can set them in correspondence $\delta \Pi^{\mu v}=\mathrm{eF}^{\mu v}$, through a constant invariant charge $\mathrm{e}$, with $\delta \mathrm{M}(\mathrm{x}, \mathrm{t})=\mathrm{eV}(\mathrm{x}, \mathrm{t}) / \mathrm{c}^{2}$ and $\delta \mathrm{P}(\mathrm{x}, \mathrm{t})=\mathrm{eA}(\mathrm{x}, \mathrm{t}) / \mathrm{c}$. The double sign for the mass variation corresponds to the two signs for electric charges, or to emission and absorption of electromagnetic energy by matter. We retrieve the minimum coupling of classical electrodynamics, $\mathrm{P}^{\mu}(\mathrm{x}, \mathrm{t})=\mathrm{p}^{\mu}+\mathrm{e} \mathrm{A}^{\mu}(\mathrm{x}, \mathrm{t}) / \mathrm{c}$, with $\mathrm{M}(\mathrm{x}, \mathrm{t}) \mathrm{c}^{2}=\mathrm{mc}^{2}+\mathrm{eV}(\mathrm{x}, \mathrm{t})$, and $\mathrm{P}(\mathrm{x}, \mathrm{t})=\mathrm{p}+\mathrm{eA}(\mathrm{x}, \mathrm{t}) / \mathrm{c}$, where the electromagnetic energy exchanged with a particle is very small with respect to its own energy e $\mathrm{A}^{\mu}(\mathrm{x}, \mathrm{t}) / \mathrm{c}=\delta \mathrm{P}^{\mu}(\mathrm{x}, \mathrm{t}) \ll \mathrm{p}^{\mu}$ (Landau,1962). The electromagnetic interaction is then directly linked to frequencies variations of the field $\varepsilon(\omega t, \mathrm{kx})$.

Relation (19) yields the relativistic Newton's equation for charged matter with the Lorentz force

\subsection{Adiabatic Invariant}

$$
\mathrm{dP} / \mathrm{dt}=-\nabla \mathrm{m}_{0} \mathrm{c}^{2} \sqrt{ }\left(1-\beta^{2}\right)+\mathrm{e}(\mathrm{E}+\mathrm{v} \times \mathrm{H} / \mathrm{c})
$$

For an almost standing wave, the relation (11) leads to first order approximation to

$$
\begin{gathered}
{\left[\partial \mathrm{U}^{2} / \partial \mathrm{t}+\nabla \cdot \mathrm{U}^{2} \mathbf{v}\right] / \mathrm{U}^{2}+\delta[\partial \Omega / \partial \mathrm{t}+\nabla \cdot \Omega \mathbf{v}] / \Omega=0} \\
\left(\partial_{v} \mathrm{~W}^{v}\right) / \mathrm{W}+\delta\left(\partial_{v} \Omega^{v}\right) / \Omega=0
\end{gathered}
$$

with $\mathrm{W}=\mathrm{W} \pm \delta \mathrm{W}=\mathrm{U}^{2}=\mathrm{u}^{2} \pm \delta \mathrm{U}^{2}$ is its total energy density and $\mathrm{W}^{v}=\mathrm{w}^{v} \pm \delta \mathrm{W}^{v}$ the four-dimensional energy density. $\Omega=\omega \pm \delta \Omega$ is the the frequency and $\Omega^{v}=(\Omega, \Omega \mathbf{v} / \mathrm{c})$, the four-dimensional frequency When we taking into account the double sign in frequency variation $\delta \Omega$ the relation (21) leads to

$$
\begin{aligned}
\mathrm{W}^{\mathrm{v}} & =\mathrm{I} \Omega^{\mathrm{v}} \\
\mathrm{W}^{\mathrm{v}} & =\mathrm{I} \omega^{\mathrm{v}} \\
\delta \mathrm{W}^{\mathrm{v}} & =\mathrm{I} \delta \Omega^{v}
\end{aligned}
$$

and

The constant $\mathrm{I}$ is an adiabatic invariant density which links the energy-momentum density $\mathrm{W}^{v}$ of the almost standing wave with the frequency $\Omega^{v}$, and their respective small variations $\delta \mathrm{W}^{v}$ and $\delta \Omega^{v}$ variations corresponding to interactions

After integrating of the total energy density $\mathrm{W}$ with respect to whole space, we get the energy $\mathrm{E}=\mathrm{mc}^{2}$ for localized matter, and the corresponding four-dimensional energy $E^{v}$ from the four-dimensional energy densities $\mathrm{W}^{v}$. The adiabatic invariant density I yields adiabatic invariant $\mathrm{H}$. We get the relations between energy and momentum of the mean field of almost monochromatic standing field

$$
\begin{gathered}
E^{v}=\mathrm{H} \Omega^{v} \\
\mathrm{E}=\mathrm{mc}^{2}=\mathrm{H} \omega \\
\mathbf{p}=\mathrm{m} \mathbf{v}=\mathrm{Hk}
\end{gathered}
$$

From (22) their small variations corresponding to absorbed or emitted interactions verify,

$$
\begin{gathered}
\mathrm{dE} \mathrm{E}^{\mathrm{v}}=\mathrm{Hd} \Omega^{\mathrm{v}} \\
\mathrm{dE}=\mathrm{c}^{2} \mathrm{dm}=\mathrm{Hd} \Omega \quad \mathrm{d} \mathbf{p}=\mathbf{v} \mathrm{dm}=\mathrm{Hd} \mathbf{k} \\
\mathrm{dE} \mathrm{E}^{\mathrm{v}}=\mathrm{d} \delta \mathrm{E}^{\mathrm{v}}(\mathrm{x}, \mathrm{t}) \\
\mathrm{dE}=\mathrm{d} \delta \mathrm{E}(\mathrm{x}, \mathrm{t})
\end{gathered}
$$




$$
\mathrm{d} \mathbf{p}=\mathrm{d} \delta \mathrm{P}(\mathrm{x}, \mathrm{t}))
$$

They are formally equivalent with quantum relations for energy-momentum of a material particle and its interactions, with the adiabatic invariant $\mathrm{H}$ corresponding to the reduced Planck's constant $\mathrm{h} / 2 \pi$.

If the standing field $\varepsilon_{0}\left(\mathrm{x}_{0}, \mathrm{t}_{0}\right)=\mathrm{u}_{0}\left(\mathrm{k}_{0} \mathrm{x}_{0}\right) \psi_{0}\left(\omega_{0} \mathrm{t}_{0}\right)$ in (4), is related to an electron, the adiabatic invariant $\mathrm{H}$ becomes identical with the Planck's constant $h$. From (23-25) we retrieve the de Broglie-Einstein's relations for matter $\mathrm{E}=\mathrm{mc}^{2}=\mathrm{h} \omega / 2 \pi$, which served at the basis of Wave Mechanics. In Quantum Mechanics, it corresponds to the second quantification, expressing that it was introduced decades after the first quantification $\mathrm{E}=\mathrm{h} v$ for light by Planck. This relation must be written $\mathrm{dE}=\mathrm{hdv}=\mathrm{c}^{2} \mathrm{dm}$ since light energy, which is emitted or absorbed by electrons, derives from variations of matter energy.

\subsection{Gravitation and General Relativity}

All above results derived from a scalar field $\varepsilon$ propagating with a constant light velocity $\mathrm{c}$ in vacuum, according to the d'Alembertian equation (4). The standing waves properties, characterized in a rest system $\left(\mathrm{x}_{0}, \mathrm{t}_{0}\right)$ by separated space and time variables $\varepsilon_{0}\left(\mathrm{x}_{0}, \mathrm{t}_{0}\right)=\mathrm{u}_{0}\left(\mathrm{k}_{0} \mathrm{x}_{0}\right) \psi_{0}\left(\omega_{0} \mathrm{t}_{0}\right)$, and a constant frequency $\omega_{0}=\mathrm{k}_{0} \mathrm{c}$, hold their stability in space when they are in uniform motion. This is expressed by the arised Lorentz transformation.

Before its application to mechanics, and more generally to special relativistic physics, the Lorentz transformation characterized the structure of Maxwell's equations in vacuum. The presence of matter, as support of electric charges, modifies locally the field properties, through induced dielectric and magnetic permeability variations, so that the field velocity propagation $\mathrm{c}$ is no longer constant, and must be written $\mathrm{C}(\mathrm{x}, \mathrm{t})$ (Elbaz, 2015). For instance, in optics, the variations are linked to a refraction index $n(x, t)=c / C(x, t)$, characteristic of the medium, which is no longer empty.

Since almost standing waves behave like localized matter, we may expect that, as a secondary effect, their energy density $\mathrm{u}^{2}=\mu \mathrm{c}^{2}$ modifies very slightly the light velocity propagation of the field, so that it remains close to its vacuum constant value, becoming $C(x, t)=c \pm \delta C(x, t)$, with $\delta C(x, t)<c$. The relation $v=\beta c$ shows that the local variations of the motion velocity $\mathrm{v}$ must follow, becoming $\mathrm{V}(\mathrm{x}, \mathrm{t})=\mathrm{v} \pm \delta \mathrm{V}(\mathrm{x}, \mathrm{t})$, with $\delta \mathrm{V}(\mathrm{x}, \mathrm{t})<<\mathrm{v}$. Consequently, the Lorentz transformation, in which the velocities $\mathrm{v}$ and $\mathrm{c}$ are constant, appears only as expressing a local approximation limit, from a more general form with varying velocities, yielding slight variations of frequency $\Omega(\mathrm{x}, \mathrm{t})=\mathrm{K}(\mathrm{x}, \mathrm{t}) \mathrm{C}(\mathrm{x}, \mathrm{t})=\omega \pm \delta \Omega(\mathrm{x}, \mathrm{t})$, and wave vector $\mathrm{K}(\mathrm{x}, \mathrm{t})=\mathrm{k} \pm \delta \mathrm{K}(\mathrm{x}, \mathrm{t})$. Then, in a rest system, space and time terms are no longer fully separated in the expression $\varepsilon_{0}\left(\mathrm{x}_{0}, \mathrm{t}_{0}\right)=\mathrm{U}_{0}\left(\mathrm{x}_{0}, \mathrm{t}_{0}\right) \Psi_{0}\left(\mathrm{x}_{0}, \mathrm{t}_{0}\right)$. The invariant interval $\mathrm{ds}^{2}=\mathrm{c}^{2} \mathrm{t}_{0}{ }^{2}=\mathrm{c}^{2} \mathrm{dt}^{2}-\mathrm{dx}^{2}$, takes then the more general local form $\mathrm{ds}^{2}=\mathrm{g}_{\mathrm{ij}} \mathrm{dx}^{\mathrm{i}} \mathrm{dx}^{\mathrm{j}}$, introducing the formalism of general relativity, and leading to all its developments and consequences.

Since the variations of all quantities are very slight in almost standing waves, as compared with their standing waves constant values, the Lorentz transformation remains locally verified when the velocity of propagation of the field $\mathrm{C}(\mathrm{x}, \mathrm{t})$ reduces to $\mathrm{c}$ at first order approximation. Inversely, dynamical properties of almost standing waves arise from those of standing waves, through variation of constants method for velocities $\mathrm{c}$ and $\mathrm{v}$, while the mass density $\mu=\mathrm{u}^{2} / \mathrm{c}^{2}$ of the standing wave limit remains unaffected in first approximation, according to (13), in the continuity equation (16) and (20)

$$
\begin{gathered}
\mathrm{d}(\mu \mathbf{V}) / \mathrm{dt}=-\nabla \mu \mathrm{C}^{2}+\nabla(\mu \mathrm{V})^{2} / 2 \mu \\
\mu \mathrm{d} \mathbf{V} / \mathrm{dt}=-\mu \nabla\left(\mathrm{C}^{2}-\mathrm{V}^{2} / 2\right) \\
\mathrm{d} \mathbf{V} / \mathrm{dt}=-\nabla\left(\mathrm{C}(\mathrm{x}, \mathrm{t})^{2}-\mathrm{V}\left(\mathrm{x}, \mathrm{t}^{2} / 2\right)\right. \\
\mathrm{d} \mathbf{V} / \mathrm{dt}_{0}=-\nabla_{0} \mathrm{C}^{2}\left(\mathrm{x}_{0}, \mathrm{t}_{0}\right)=-\nabla_{0} \Phi\left(\mathrm{x}_{0}, \mathrm{t}_{0}\right) .
\end{gathered}
$$

The acceleration $\mathrm{d} \mathbf{V} / \mathrm{dt}$ of an almost standing wave, either at rest or in motion, is locally independent of its energy density, equivalent to mass density of matter. This characterizes gravitation interaction (Elbaz, 2015). The gravitational potential $\Phi(\mathrm{x}, \mathrm{t})=\mathrm{C}^{2}(\mathrm{x}, \mathrm{t})$ is formally identical with the square of the local velocity of propagation of the field.

The relations (26) are consistent with physical origin of gravitation, exposed in 1912 by Einstein, in a preliminary article on general relativity of 1915, on Light velocity and static gravitation field, (Einstein,1912) He established the equation $\Delta \mathrm{c}=0$ in vacuum, generalizing the Poisson equation $\Delta \Phi=0$ for the gravitation potential.

\section{Incompleteness of General Relativity}

Since it derived gravitation and quantification of matter and interactions through adiabatic process, the scalar field following Einstein's program brings some elements to circumvent the two difficulties actualized by the 
detection of gravitational waves: incompleteness of General Relativity theory with regard to its quantification, and coexistence of field and particle.

\subsection{General Relativity and Quantum Theory}

With regard to quantification, the scalar field $\varepsilon$ enlightens the lasting theoretical and experimental difficulties encountered by General Relativity.

From a theoretical point of view, it shows that the respective physical natures of gravitation and electromagnetic interactions are separated: gravitation results from local variations of speed of the field, while electromagnetic interaction and Planck's constant yielding quantification, result from local variations of frequencies.

Such physical difference brings justification encountered by gravitation for its quantification after one century of efforts, while electromagnetic interaction was closely linked to Planck's constant since its introduction.

The close physical relation between gravitation interaction and local variations of light velocity appeared implicitly at establishment of general relativity theory, beyond the occultation of its electromagnetic nature, and even of its physical meaning as velocity. As an extension of special relativity, the general relativity theory involved locally variable $\mathrm{g}^{\mathrm{ij}}$ coefficients for space-time metric of universe, instead of constant coefficients: velocity $\mathrm{c}$ for light, and velocity $\mathrm{v}$ for matter. In the space-time metric, the pillar term $\mathrm{c}$ becomes then $\mathrm{C}(\mathrm{x}, \mathrm{t})$. The velocity of light varies following action of matter like in geometrical optics. The refraction index $\mathrm{n}$ fixes then the geometry of light rays: strait lines for $\mathrm{n}$ constant in homogenous medium, and curved lines when $\mathrm{n}$ varies continuously. In optics light and its velocity $\mathrm{c}$ are of electromagnetic nature. Such a physical property was abandoned at establishment of special relativity theory, following the extension of kinematic properties of charged matter, described by Maxwell's equations, to neutral matter described by mechanics (Einstein 1905). The term c remained only a mathematical coefficient in space-time metric of universe.

The close physical relation between electromagnetic interaction and quantification, both resulting from local variations of frequencies, was explicit from the beginning, when Planck introduced his constant as a link between energies of oscillations of electrons and light radiations, and then in exchange of electromagnetic energies between localized matter and extended wave light. It was established in a wave-particle dual framework, before the advent of special relativity. Electrons as point-like particles with constant mass, were geometrically heterogeneous with regard to extended and continue radiation waves. In order to remain in a homogeneous field framework, Planck assimilated electrons to oscillators of electromagnetic field in1900. Thus, he emphasized that his introduced constant was a specific property of oscillating extended field energy. But it was only implicit. In 1905, with the photoelectric effect, Einstein initiated the opposite current by showing that the field energy was constituted of particles. In appreciation of its importance, the Nobel Prize in 1921 awarded him for this discovery, and not for general relativity theory. It led to the present standard model. Consequently, when in1911 Ehrenfest emphasized the role of oscillations, his works did not raise up enough interest. He established that the Planck's constant was an adiabatic invariant: a quantity which remained constant during energies transformations inside a cavity without outside exchanges (Ehrenfest, 1911), (Navarro, 2006). Until now, the Planck's constant is generally admitted as fundamental. A possible more profound physical signification, like a formal analogy with an adiabatic invariant, is mostly ignored. It is considered as less important than general its applications to all energies, at one and the same time for extended field interactions and for localized particles.

The detection of gravitational waves showed off two main results. It emphasized that the Planck's constant does not apply directly to the gravitational field, which is not quantized, and then not associated with mediating particles. It actualizes the problem of coexistence of particle and field, raised by Einstein about the incompleteness of the General Relativity equations.

\subsection{General Relativity and Coexistence of Particle and Field}

Einstein's comparing their left side to « one wing of a building made of fine marble"appears supported at different titles.

In one hand, the accuracy of Einstein's equations, lying essentially in the left side, has been improved by the higher precision required to detect the minuscule effect of gravitational waves with very small amplitude. Such a precision is consistent with the highest level of experimental precision reached for measurements of frequencies allowed by the international standard of time. In another hand, the gravity field is described by partial differential equations which were privileged by Einstein in his program. 
From the deriving $\varepsilon$ field we find an explanation for the minuscule effect, not only of gravitational waves, but of gravitation itself with regard to electromagnetic interaction: the gravitational waves represent only a fourth approximation of field-energy.

In order to fix it, we notice that isolated particles of matter, corresponding to as standing waves, are characterized by $\omega$ constant frequencies. They represent a first level of approximation for field-energy. Electromagnetic interactions, linked to slight local variations of frequencies $\delta \Omega(x, t)<<\omega$ correspond to second order approximations. In both cases the field-energy propagates at light velocity $\mathrm{c}$ in vacuum. Gravitation, linked to slight local variations of field velocity $\delta \mathrm{C}(\mathrm{x}, \mathrm{t})<<\mathrm{c}$ represents a third level of approximation. Gravitational waves, as variations of gravitation energy, correspond then only to a fourth level of approximation for field-energy.

The right side of the equations, compared by Einstein's to "one wing of which is built of low grade wood" appears supported at different titles.

By comparison with gravity field, the mass-energy density is described with a much lower precision, particularly because the international standard of mass is still defined through a macroscopic body.

Nevertheless matter plays an important role of in the right side by determining the properties of gravitational field in the left side, without being directly affected in return. Notwithstanding, the gravitational waves propagate at light velocity $\mathrm{c}$ in vacuum. It is theoretically obtained by cancelling the right side, outside matter, either because matter-energy is geometrically well localized like in particles, or it no longer present like after black holes collapse. This raises some questions: since gravity is closely linked to matter, while gravitational waves propagate in vacuum, should gravity exist without matter? And then, what should be the physical nature of the medium of propagation: a new kind of ether deprived of electromagnetic properties as Einstein questioned (Einstein, 1920)?

\section{Conclusion}

The above features emphasize the pre-eminence of the field with regard to matter, as posited by Einstein in his program.

The following $\varepsilon$ scalar field offers means to circumvent difficulties raised about the incompleteness of general relativity theory with regard to the problem of coexistence of particle and field and quantum gravitation.

It derives particles of matter from standing waves which singularizes with two main properties: stable localization in space, behaving as point-like in the geometrical optics approximation for very high frequencies undetectable experimentally, and holding in motion with a constant speed strictly inferior to speed of light.

It shows that quantification does not concern especially directly gravitation since it derives from variations of speed of field, but more directly electromagnetic interaction which is linked to variations of frequency like the Planck's constant.

More generally the $\varepsilon$ scalar field allows a little deeper insight into foundations of relativity theories. The standing waves exhibits kinematical and dynamical properties are formally identical with matter.

They yield the special relativity framework with the Lorentz transformation and the space-time metric of universe with constant coefficients, and the general relativity framework when they vary locally.

It supplements them by introducing a space-like $u$ amplitude function, to describe the space extension of a standing field, introducing the energy density $\mathrm{u}^{2}$ and the energy-momentum conservation law and the least action principle, usually admitted as fundamental.

It forms a common and homogenous framework for classical and quantum physics and for the coexistence of matter particle and interactions field, paving the way toward a unified physical approach of universe properties, according to Einstein's program.

\section{References}

Abbott, B. P., Abbott, R., Abbott, T. D., Abernathy, M. R., Acernese, F., Ackley, K., ... \& Adya, V. B. (2016). Observation of gravitational waves from a binary black hole merger. Physical review letters, 116(6), 061102. https://doi.org/10.1103/PhysRevLett.116.061102061102

Dalibard, J. (2016). Atomes et lumière en équilibre thermique: de l'argument d'Einstein aux mélasses optiques. Paris: College de France.

Ehrenfest, P. (1911). Welchezüge der lichquantenhypothese spielen in der theorie der Wärmestrahlung eine wesentliche rolle? Ann Phys, 35, 91-118; 
Einstein, A. (1912). Lichtgeschwindigkeit und statik des Gravitationsfeldes. Annalen der Physik, 343(7), 355-369.

Einstein, A. (1916). Strahlungs-emission und-absorption nach der Quantentheorie. Verh. Deutsch. Phys. Gesell., $18,318-323$.

Einstein, A. (1920). Aether and the Theory of Relativity: University of Leyden.

Einstein, A. (1929). Einstein's Theory of Relativity. conference

Einstein, A. (1936) Physics and reality. Journal of Franklin, Institute.

Einstein, A. (1949). Philosopher, Scientist. London: Cambridge University Press.

Einstein, A., \& Infeld, L. (1938). The Evolution of Physics (pp. 228-232). Cambridge: Cambridge Univ Press.

Einstein, A., \& Rosen, N. (January 1937). On gravitational waves. Journal of the Franklin Institute, 223(1), $43-$ 54.

Elbaz, C. (1984). L'onde stationnaire et la transformation de Lorentz. Comptes-rendus des séances de l'Académie des sciences. Série 2, Mécanique-physique, chimie, sciences de l'univers, sciences de la terre, 298(13), 543-546.

Elbaz, C. (2010). Dynamic properties of almost monochromatic standing waves. Asymptotic Analysis, 68(1-2), $77-88$.

Elbaz, C. (2012). Discrete and Continuous Dynamical Systems. A.I.M.S, Series B, 17, 835-849.

Elbaz, C. (2014). Wave-particle duality in Einstein-de Broglie programs. Journal of Modern Physics, 5(18), 2192-2199. http://dx.doi.org/10.4236/jmp.2014.518213

Elbaz, C. (2015). Gravitation and Electromagnetism Conciliated Following Einstein's Program. Journal of Modern Physics, 6(05), 660-669. http://dx.doi.org/10.4236/jmp.2015.65072

Elbaz, C. (2015). On Einstein's Program and Quantum Mechanics. Applied Physics Research, 7(6), 126. http://dx.doi.org/10.5539/apr.v7n6p126

Elbaz, C. (2016). On Einstein's Program and Quantum Double Slit Experiment. Studies in Engineering and Technology, 3(1), 91-100. http://dx.doi.org/10.11114/set.v3i1.1730

Elbaz, C. L. A. U. D. E. (2013). Sur les programmes d"e Albert Einstein et de Louis de Broglie. Une contribution. In Annales Fondation Louis de Broglie (Vol. 38, pp. 195-217).

Jefferts, S. R., Heavner, T. P., Barlow, S. E., \& Ashby, N. (2015). Comment on "Ramsey spectroscopy, matter-wave interferometry, and the microwave-lensing frequency shift". Physical Review A, 91(6), 067601.

Landau, L., \& Lifchitz, E. (1960). Mechanics. Pergamon.

Landau, L., \& Lifchitz, E. (1962). The Classical theory of fields. Pergamon.

Navarro, L., \& Pérez, E. (2006). Paul Ehrenfest: The genesis of the adiabatic hypothesis, 1911-1914. Archive for History of Exact Sciences, 60(2), 209-267.

\section{Copyrights}

Copyright for this article is retained by the author(s), with first publication rights granted to the journal.

This is an open-access article distributed under the terms and conditions of the Creative Commons Attribution license (http://creativecommons.org/licenses/by/4.0/). 